\title{
Der Rosa-Luxemburg-Platz in Radebeul
}

\author{
Janos Fath, Manuela Jacobs, Sabrina Wenck
}

$\mathrm{D}$ er heutige Rosa-Luxemburg-Platz (früher Königsplatz) liegt im Zentrum des Radebeuler Stadtteils Niederlößnitz. Die Weinberge des Denkmalschutzgebietes »Historische Weinbergslandschaft Radebeul« bilden die Kulisse für die als Vorplatz für das ehemalige Rathaus Niederlößnitz angelegte Grünfläche. Diese reiht sich in eine Kette von Schmuckplätzen, wie z.B. Fontainenplatz und Zillerplatz, ein, die um die Wende zum 20. Jahrhundert in den Ortschaften Nieder- und Oberlößnitz auf Initiative des »Verschönerungsvereins für die Lößnitz« sowie der Gebrüder Moritz und Gustav Ziller, Inhaber eines ortsansässigen Bauunternehmens, entstanden. Die Schmuckplätze sollten auch in der Lößnitz »eine Wohltat für das Gemeinwohl « ${ }^{1}$ sein und zudem die von den Gebrüdern Ziller neu erschlossenen Villenviertel attraktiver für potentielle Bewohner machen. Sie entstanden in unterschiedlichen Grundrissen und Ausformungen an Kreuzungspunkten der Villengebiete und bildeten mit den umgebenden Grundstücken eine gestalterische Einheit. ${ }^{2}$ Im Wintersemester 2017/18 wurde im Rahmen der Lehrveranstaltung Gartendenkmalpflege im Bachelor Landschaftsarchitektur an der TU Dresden eine gartendenkmalpflegerische Zielstellung für den Rosa-Luxemburg-Platz erarbeitet. Die Besonderheit war hierbei, dass der Platz nicht unter Denkmalsschutz steht und eine grundlegende Umgestaltung der Anlage, die im Jahr 2018 realisiert wurde, unmittelbar bevorstand. Aus diesem Grund bezieht sich die folgende Bestandsbeschreibung auf den
Zustand Ende 2017 und damit auf das Aussehen des Platzes unmittelbar vor seiner Neugestaltung (Abb. 1).

Der Platz besitzt eine rechteckige Grundform (ca. 65 x $50 \mathrm{~m}$ ). Die ihn begrenzenden Straßen werden zum großen Teil von Straßenbäumen (Tilia cordata) gesäumt. Die Randbereiche des Platzes werden von raumprägenden Großgehölzen und immergrünen Gehölzbereichen umschlossen. Im mittleren Bereich sind Strauch- und Staudenpflanzungen sowie Rasenflächen zu finden. Die Anlage ist durch diagonal und bogenförmig verlaufende wassergebundene Wege erschlossen (Abb. 2). An der Südwest-Seite des Platzes befindet sich ein etwa $140 \mathrm{~m}^{2}$ großes Podest mit einem Mahnmal für die Opfer des Faschismus. Das Mahnmal - gestaltet als Gedenktafel aus geschliffenem Granit auf einer verputzten Betonstele - wird von zwei Pflanzschalen flankiert (Abb. 3)

Der vielfältig genutzte Rosa-LuxemburgPlatz befand sich Ende 2017 in einem schlechten Erhaltungszustand. Die auf ein Minimum reduzierte Pflege zeigte sich vor allem am Zustand der Wege (stellenweise freiliegende Tragschichten), an den vernachlässigten Ausstattungselementen und der fehlenden Sauberkeit.

Die historische Entwicklung des Platzes lässt sich in fünf Phasen einteilen. Die Abgrenzung der Phasen voneinander ergab sich durch die deutlichen Unterschiede in der Gestaltung, der Gesamtkonzeption, der Nutzung sowie der sozialen und politischen Bedeutung des Platzes. 


\section{Der Rosa-Luxemburg-Platz in Radebeul}

\section{Phase 1: Entstehung der Platzsituation (bis 1895)}

Die Fläche des heutigen Rosa-LuxemburgPlatzes ist bereits 1875 im Ortsbauplan als kreisrunder Platz eingezeichnet, obwohl zu dieser Zeit die Umgebung des Platzes noch unbebaut und in erster Linie von landwirtschaftlicher Nutzung bestimmt war. Durch die Reblauskatastrophe Anfang der 188oer Jahre wurden riesige Flächen in der Lößnitz für den Weinanbau unbrauchbar. Gleichzeitig waren die Ortschaften Ober- und Niederlößnitz im Begriff, sich zu gefragten Villenvororten der nahe gelegenen Residenzstadt Dresden zu entwickeln. ${ }^{3}$ Diese Umstände wussten die Gebrüder Ziller sinnvoll miteinander zu verbinden: Sie trugen entscheidend zum Bauboom in diesen »Gründerjahren der Lößnitz« bei, indem sie brachliegende Flurstücke erwarben, um dort (Miet-)Villen zu errichten. Auf diese Art entstanden neue Straßenzüge und ganze Villenviertel, die Radebeul, das "Sächsische Nizza«, bis heute prägen. ${ }^{4}$ Die Fläche des Rosa-Luxemburg-Platzes liegt an einer solchen Villenstraße, die 1885 zu Ehren von König Albert von Sachsen (1828-1902) den Namen Königstraße erhielt. Der Platz wurde in diesem Zusammenhang Königsplatz benannt. Schon wenige Jahre später begann seine erste Gestaltung durch den Gärtner Alexander Steffen 5 . Wie der Platz zu dieser Zeit genau aussah, ließ sich aufgrund fehlender Quellen nicht ermitteln.

Der 1890 von der Gemeinde Niederlößnitz gefasste Entschluss, an der Nordseite des Königsplatzes ein repräsentatives Rathaus zu errichten, spielte eine entscheidende Rolle bei der Entwicklung des Königsplatzes hin zu einem gärtnerisch gestalteten Schmuckplatz. Nachdem das passende Flur-

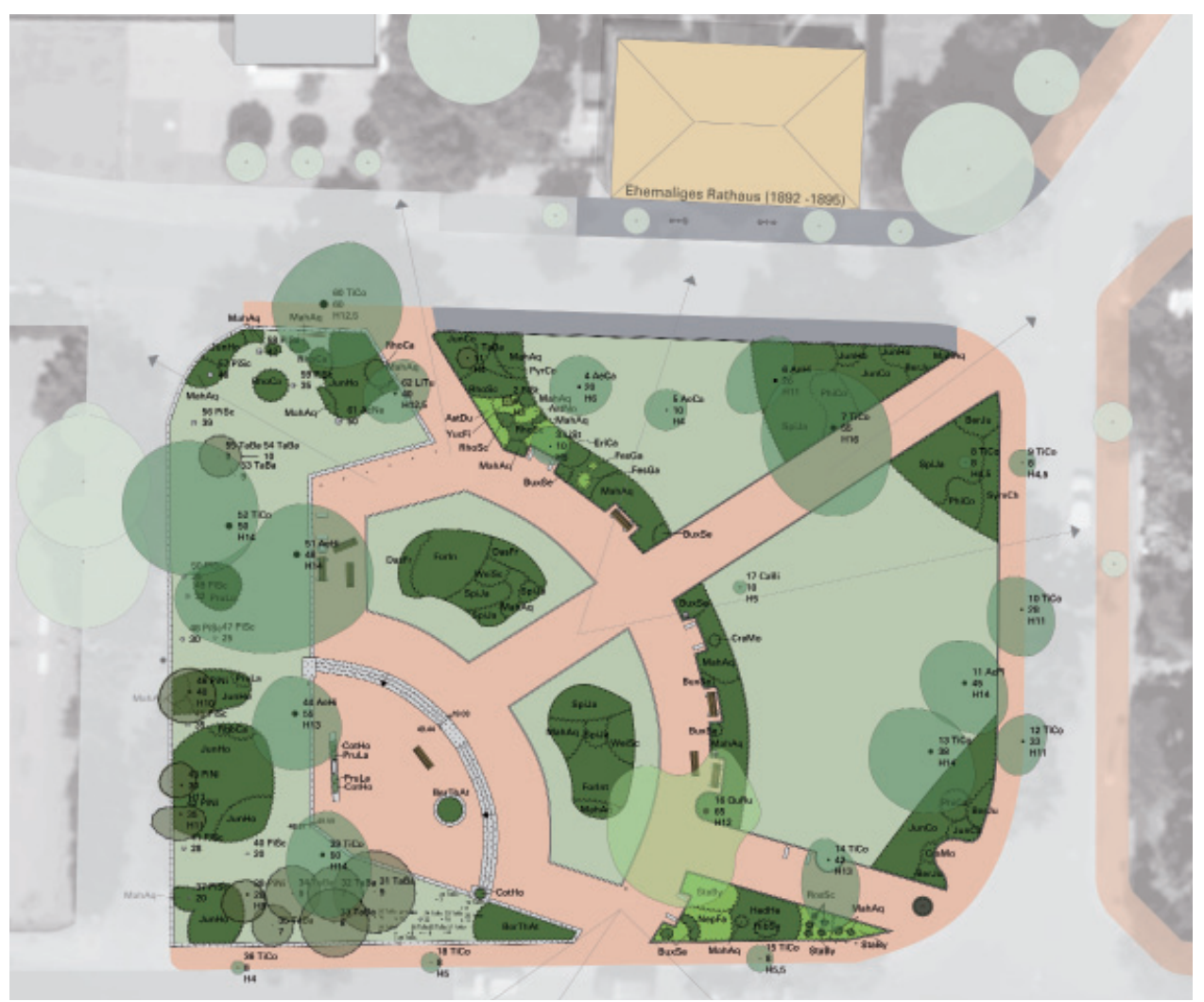

1) Bestandsplan Rosa-Luxemburg-Platz Radebeul, eigener Plan 2017, genordet. 


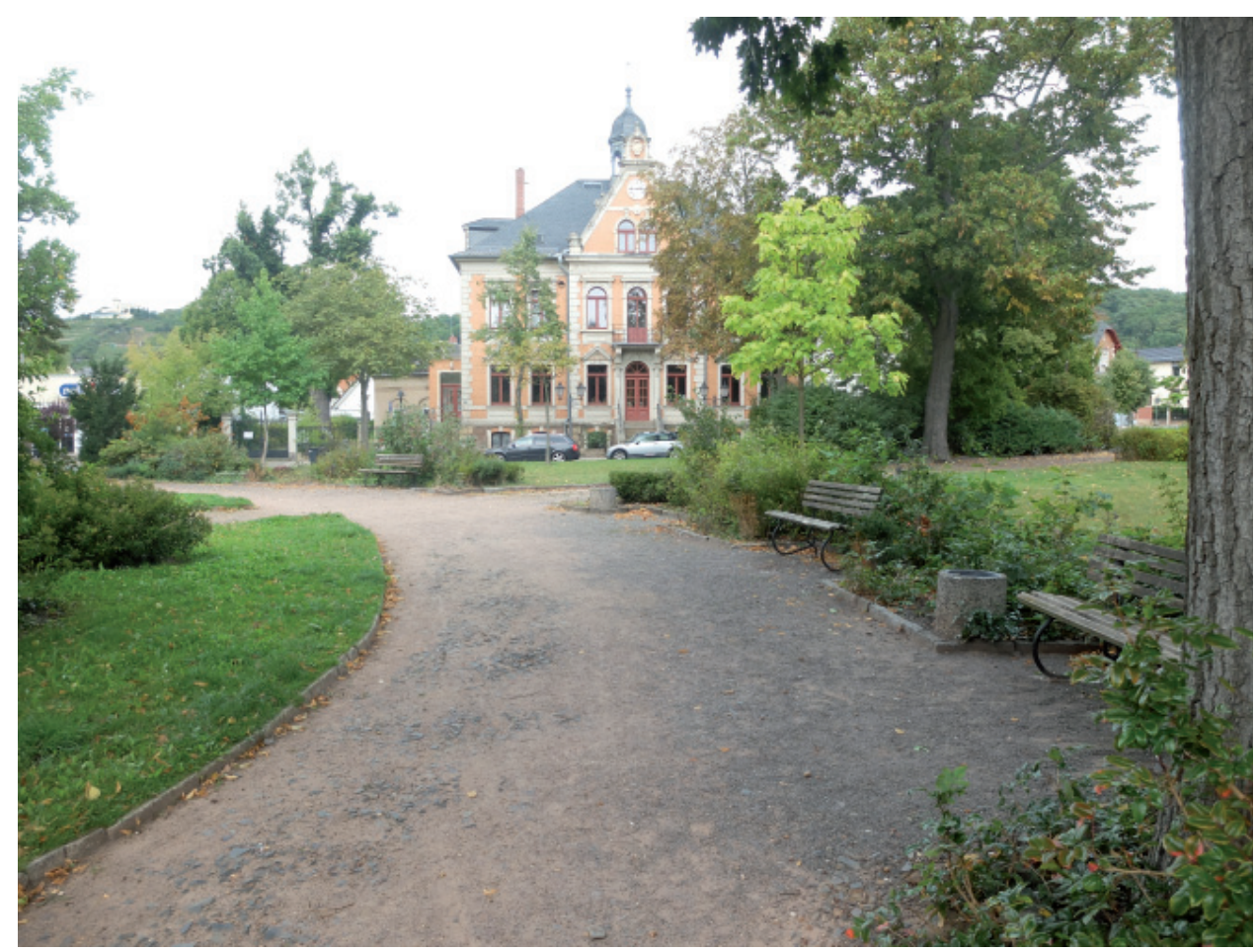

2| Blick nach Norden zum ehemaligen Rathaus, Fotografie: Marlen Hößelbarth, 2017.

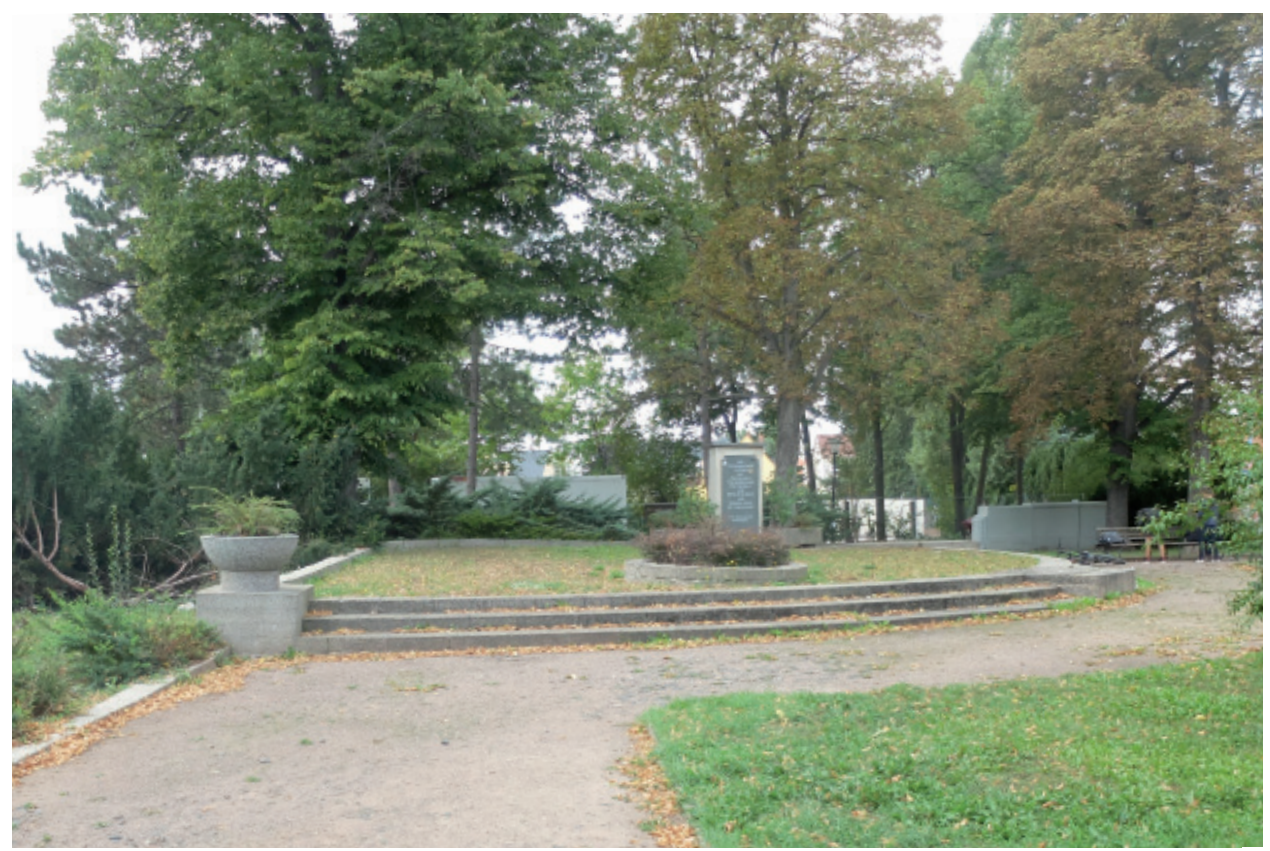

3 | Blick nach Westen zum Podest mit dem Mahmal für die Opfer des Faschismus, Fotografie: Marlen Hößelbarth, 2017. 
stück mit Genehmigung der zuständigen Amtshauptmannschaft Dresden-Neustadt erworben war ${ }^{6}$, konnte das Rathausgebäude unter Leitung des ortsansässigen Architekten Adolf Neumann rasch fertiggestellt werden. Die Einweihung fand am 4. April 1895 statt. ${ }^{7}$ Die Entwicklung des Platzes und seiner Gartenanlage sind ab diesem Zeitpunkt eng mit der des Rathausgebäudes und dessen jeweiliger Nutzung verbunden. Aus dieser Phase haben sich keine baulichen oder vegetabilen Elemente erhalten. Allerdings wurde die rechteckige Grundstruktur des Platzes seither nicht verändert und war für die weitere städtebauliche Entwicklung der Platzumgebung maßgeblich.

\section{Phase 2: Ausgestaltung zum Schmuck- platz (1895-1949)}

Zum Ende des 19. Jahrhunderts wurde der in der Oberlößnitz ansässige Landschaftsgärtner Gustav Pietzsch durch die Gemeinde mit der »Herstellung von Gärtnerischen Anlagen auf dem Platz vor dem Rathaus« auf einer Fläche von $2650 \mathrm{~m}^{2}$ betraut. ${ }^{8}$ Der Verschönerungsverein unterstützte das Vorhaben finanziell mit einer Summe von 300 Mark. ${ }^{9}$ Die Arbeiten begannen Anfang August 1895 mit der Herstellung eines Wasserbassins im Zentrum des Platzes..$^{10}$ Abbildung 4 zeigt die neu angelegte Wegeführung mit dem Wasserbassin in der Mitte und die Platzierung der Straßenbäume, die rund um den Platz und entlang der anliegenden Straßen gepflanzt wurden. Die Erdarbeiten waren bereits im September 1895 abgeschlossen. Die Pflanzungen sowie die Rasenflächen fasste man kurz darauf mit niedrigen Jägerzäunen ein. 1899 beschloss die Gemeinde die »Aufstellung von Bänken in den Königsplatzanlagen « ${ }^{11}$. Der Gärtner Pietzsch wurde bis 1903 mit der Pflege und Instandhaltung des Königsplatzes beauftragt, danach unterhielt die Gemeinde Niederlößnitz die Anlage selbst. Neben den Bänken wurde die Ausstattung des Platzes um eine Beleuchtung in Form von zwei Kandelabern ergänzt. Es befanden sich insgesamt 14 Bänke auf dem Königsplatz: zwölf Bänke in den halbrunden Sitznischen des Platzes und zwei weitere an den gegenüberliegenden Seiten des Wasserbassins. Zur Ausstattung gehörte außerdem eine Litfaßsäule an der südöstlichen Platzseite. Einen guten Eindruck der damaligen Platzgestaltung vermittelt eine zeitgenössische Postkarte von 1905 (Abb.5).

1915 sollte im Zuge der Sanierung des undicht gewordenen Bassins auch dessen Einfriedung verbessert und die Jägerzäune durch ein modernes Promenadengeländer ersetzt werden..$^{12}$ Das Wasserbassin war zeitweise mit einer Fontäne ausgestattet. In den Folgejahren wurden keine gravierenden Veränderungen an der Anlage vorgenommen. Als 1938 durch Straßenumbaumaßnahmen an der Kreuzung Kaiserstraße / Königstraße das Kaiser-Wilhelm-Denkmal einen neuen Platz benötigte, stellte die Gemeinde es auf dem Königsplatz auf. ${ }^{13}$ Nach der Machtergreifung der Nationalsozialisten benannte man den Platz im April 1939 in Ludendorff-Platz um. ${ }^{14}$ Das Kaiser-Wilhelm-Denkmal wurde 1945 nach Kriegsende abgebaut und der Platz erhielt im Zuge der Entnazifizierung den Namen Rosa-Luxemburg-Platz. ${ }^{15}$

Aus der Schmuckplatz-Phase sind heute keine baulichen Elemente mehr erkennbar. Allerdings sind einige Gehölze aus dieser Zeit erhalten: Die Standorte der Winter-Linden (Tilia cordata) lassen sich jeweils rechts und links den ehemaligen Sitznischen zuordnen. Eine eindrucksvolle und raumprägende Rot-Eiche (Quercus rubra) stammt ebenfalls aus dieser Zeit. Ein altersgleiches Äquivalent ist links neben dem ehemaligen Rathausgebäude zu finden. Zwei Rosskastanien (Aesculus hippocastanum) sind ihrem Alter und Standort nach ebenfalls in diese Phase einzuordnen.

\section{Phase 3: Ausbau zum Gedenkort mit VVN-Mahnmal (1949-1980)}

Mit der Umbenennung in Rosa-Luxemburg-Platz erhielt der Platz eine politische Bedeutung. Die VVN-Ortsgruppe Radebeul 


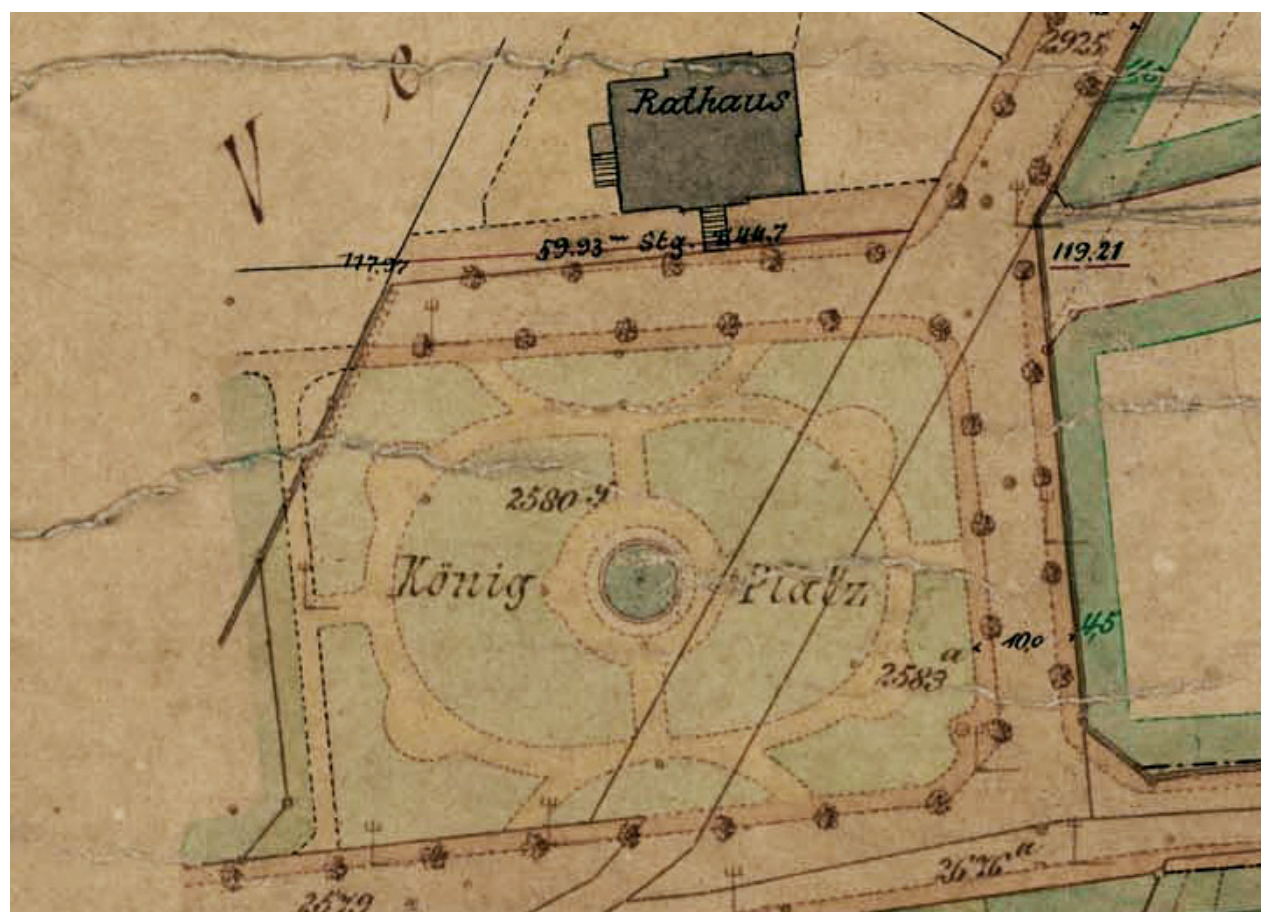

4 | Ausschnitt aus: Bebauungsplan der Flur Niederloessnitz. Section II., kolorierte Handzeichnung, 1902-1904 (Stadtarchiv Radebeul, k33).

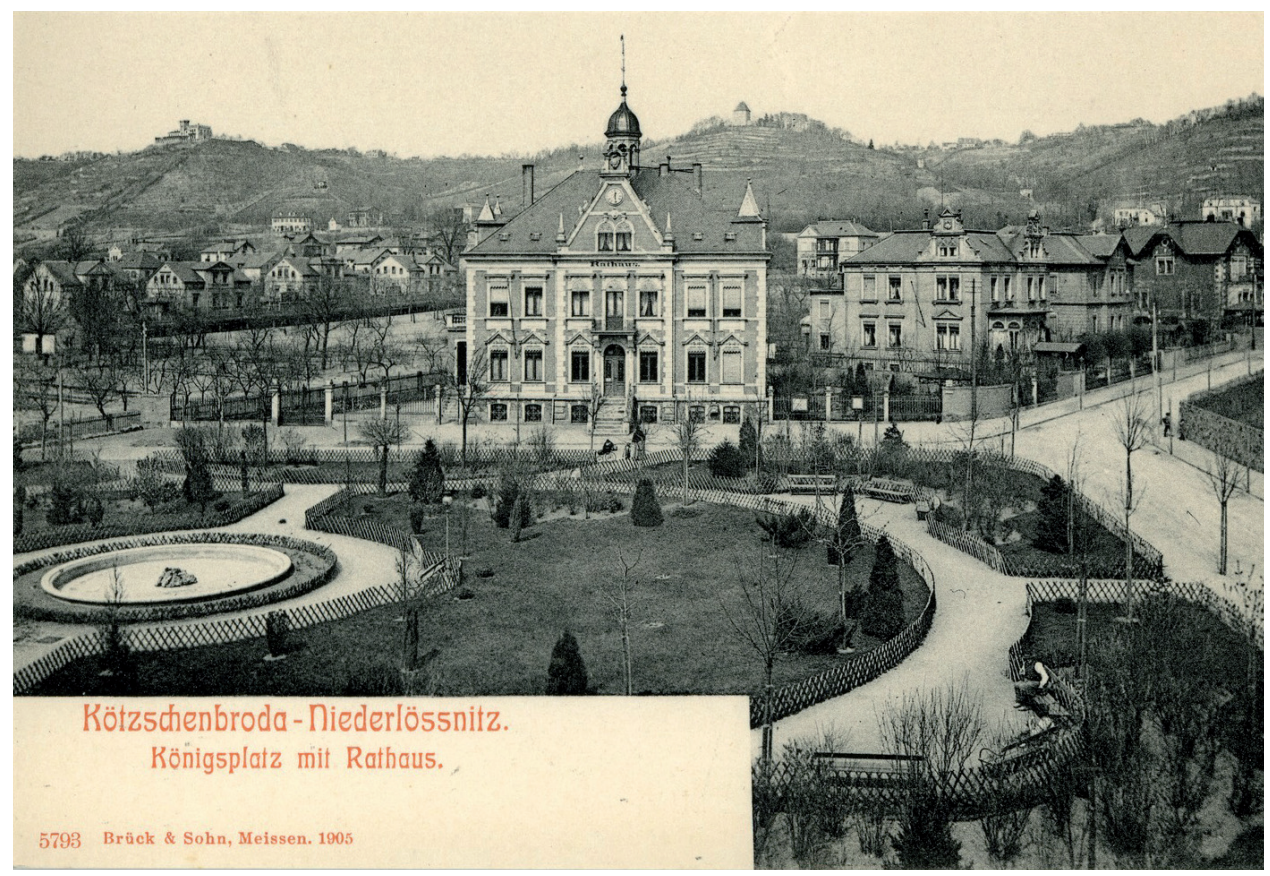

5 | Blick auf den Königsplatz mit Rathaus und Villen an der Langenstraße (Dr.-Rudolf-Friedrichs-Straße), Postkarte, Brück \& Sohn Kunstverlag Meißen, 1905. 


\section{Der Rosa-Luxemburg-Platz in Radebeul}

beantragte im September 1949 beim zuständigen Bauaufsichtsamt Radebeul die Aufstellung eines Mahnmals für die Opfer des Faschismus. Der Bau des Mahnmals wurde im Oktober 1949 genehmigt und der Gedenkstein mit der Aufschrift:

»Den unsterblichen Opfern des Faschismus und Kämpfern für Freiheit und Menschlichkeit. Wir werden euch nie vergessen! VVN Ortsgruppe Radebeul«

am 15. April 1950 enthüllt. ${ }^{16}$ Aus den Unterlagen der Bauaufsicht geht hervor, dass neben der Errichtung des Mahnmals eine Umgestaltung der umgebenden Gartenanlage geplant und genehmigt wurde. ${ }^{17}$

Mit der Entwicklung zum Gedenkort veränderte sich der Grundcharakter des Platzes (Abb. 6). Das Wasserbassin und die mittigen Rasenflächen wurden entfernt, so dass an ihrer Stelle ein Appellplatz entstehen konnte. Der Standort des Mahnmals an der Westseite zwischen zwei Rosskastanien sorgte für eine Neuausrichtung der Blickbeziehungen. Das Rathaus war nicht länger Hauptbezugspunkt. Durch seine Umnutzung zum Kindergarten ab $1953^{18}$ war ein repräsentativer Vorplatz zudem nicht mehr nötig. Die axialsymmetrische Gestaltung des Schmuckplatzes blieb dennoch teilweise erhalten und ebenso große Teile des Vegetationsbestandes. Auf der Westseite pflanzte man als Hintergrund und Rahmen für das Mahnmal zahlreiche immergrüne Gehölze. Das Mahnmal selbst stand auf einem Betonsockel mit drei Stufen (Abb. 7). Die Fläche vor dem Mahnmal wurde mehrfach verändert. $\mathrm{Zu}$ nächst befand sich dort ein quadratisches Schmuckbeet, später eine größere Rasenfläche mit einem umlaufenden Weg aus Betonpflaster. Zur Ausstattung des Platzes gehörten außerdem eine Reihe Fahnenmasten auf der rechten Seite des Mahnmals sowie einige Sitzbänke.

1973 wurde die benachbarte Kaufhalle eröffnet. Damit veränderten sich die Anforderungen an den Platz und es folgte eine Um-

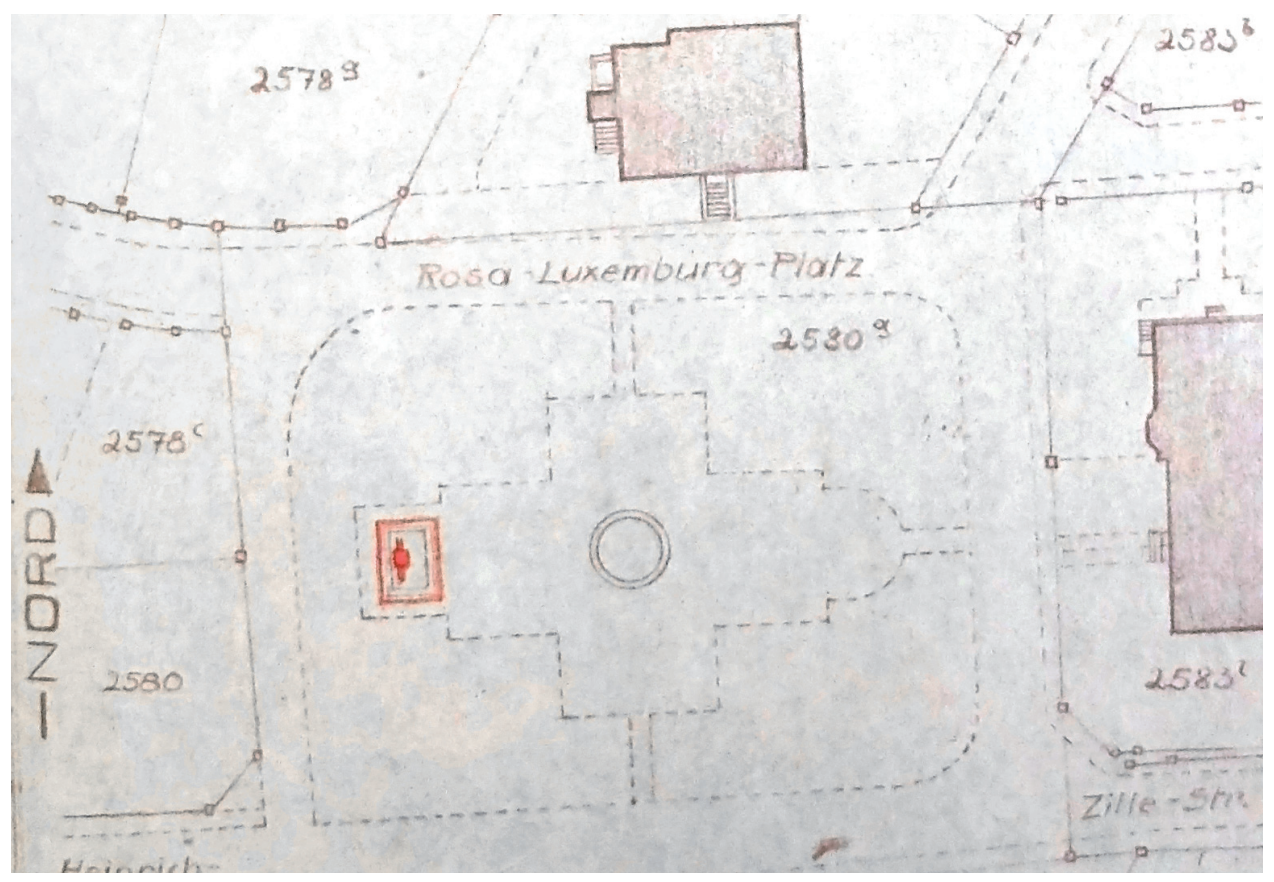

6| Ausschnitt aus: Bauantrag der VVN-Ortsgruppe von 1949. Planung für Appellplatz mit VVN-Denkmal (Stadtarchiv Radebeul, S 11-21 Gedenkstätten der Radebeuler Arbeiterbewegung). 


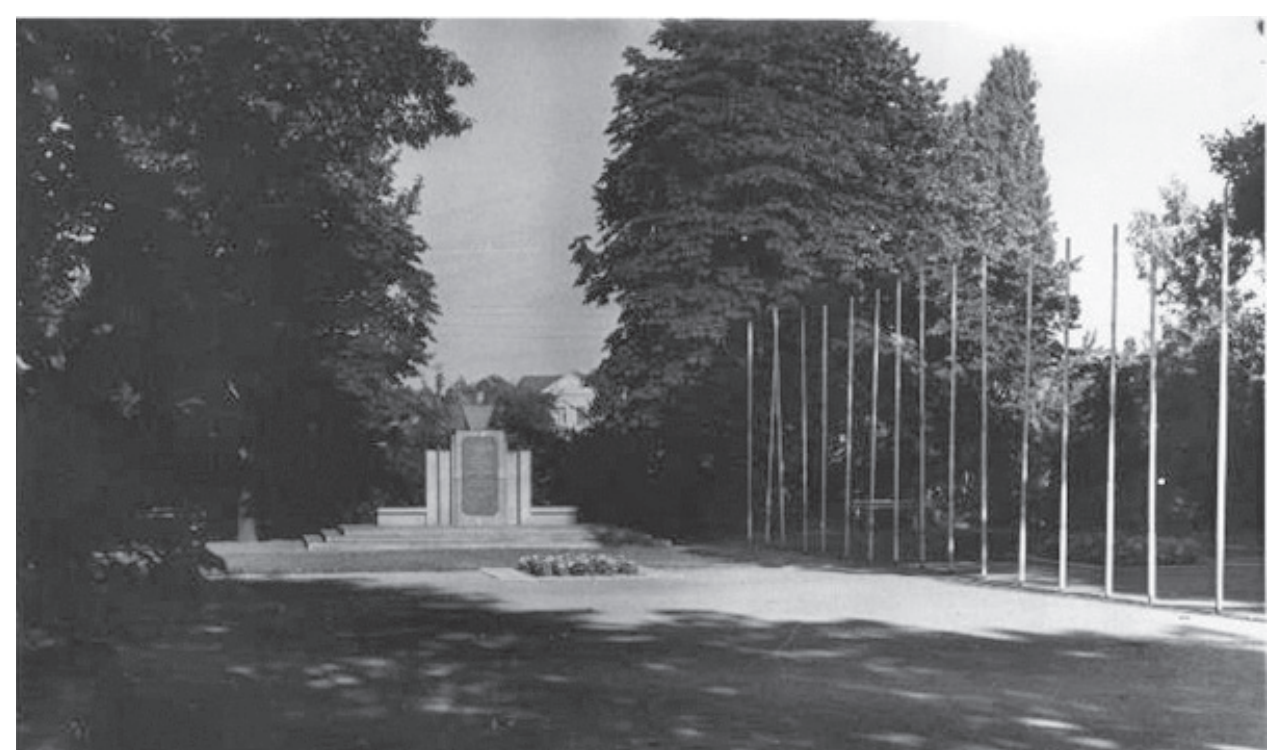

7 | Gedenkstätte für die Opfer des Faschismus auf dem Rosa-Luxemburg-Platz, Fotografie, 195oer Jahre (Stadtarchiv Radebeul, Bild-Nr. 3009).

gestaltung, die wahrscheinlich zeitgleich mit der Eröffnung der Kaufhalle abgeschlossen wurde. Man passte die Wegeführung an, so dass die Kaufhalle ohne Umwege erreichbar war. Es entstand eine halbrunde, asymmetrische Platzform, wobei man den vorhandenen Baumbestand weitgehend integrierte (Abb. 8). Der Charakter des Appellplatzes blieb dabei erhalten, ebenso verblieb das Mahnmal an seinem Standort. Der Park erhielt mehr Aufenthaltsqualität durch die Aufstellung neuer Bänke in Nischen, die mit Schmuckpflanzungen umgeben waren (Abb. 9). Von wem der zugrunde liegende Entwurf stammte, konnte nicht ermittelt werden.

\section{Phase 4: Umgestaltung mit Aufstellung des Mahnmals von Johannes Peschel (1980 - 1990)}

Im August 1978 beschloss der Rat der Stadt Radebeul Maßnahmen zur Neu- bzw. Umgestaltung des Ehrenmals am Rosa-Luxemburg-Platz, die bis zum 35. Jahrestag der Befreiung im Mai 1980 abgeschlossen sein sollten. ${ }^{19}$ Nachdem diese Planungen zunächst im Sande verliefen, wurde der Dresd- ner Bildhauer Johannes Peschel im September 1980 mit dem Entwurf eines neuen Ehrenmals beauftragt. ${ }^{20}$ Peschel entwickelte im Kollektiv mit dem Landschaftsarchitekten Günther Kretzschmar ein Gestaltungskonzept, dass die bereits bestehende Wegeführung aufnahm und ergänzte (Abb. 10). Im Erläuterungsbericht für die Neugestaltung heißt es: »Das [...] Denkmal lässt inhaltliche und formale Fragen offen und entspricht auch in seiner standörtlichen Beziehung nicht mehr der vor einigen Jahren durchgeführten Umgestaltung der Parkanlage, insbesondere der Wegeführung. $^{21}$ Der Standort des neuen Mahnmals lag in Beziehung zur Durchwegung. Die Halbkreisstruktur des Platzes wurde aufgenommen und ein Podest geplant, auf dem die »Figurengruppe im Widerstand ${ }^{22}$ und eine Spruchwand aus Sandstein aufgestellt werden sollten. Die Spruchwand setzte sich aus 15 Einzelblöcken zusammen und trug die Aufschrift:

»Ehrendes Gedenken den Kämpfern gegen Faschismus und imperialistischen Krieg" 


\section{Der Rosa-Luxemburg-Platz in Radebeul}

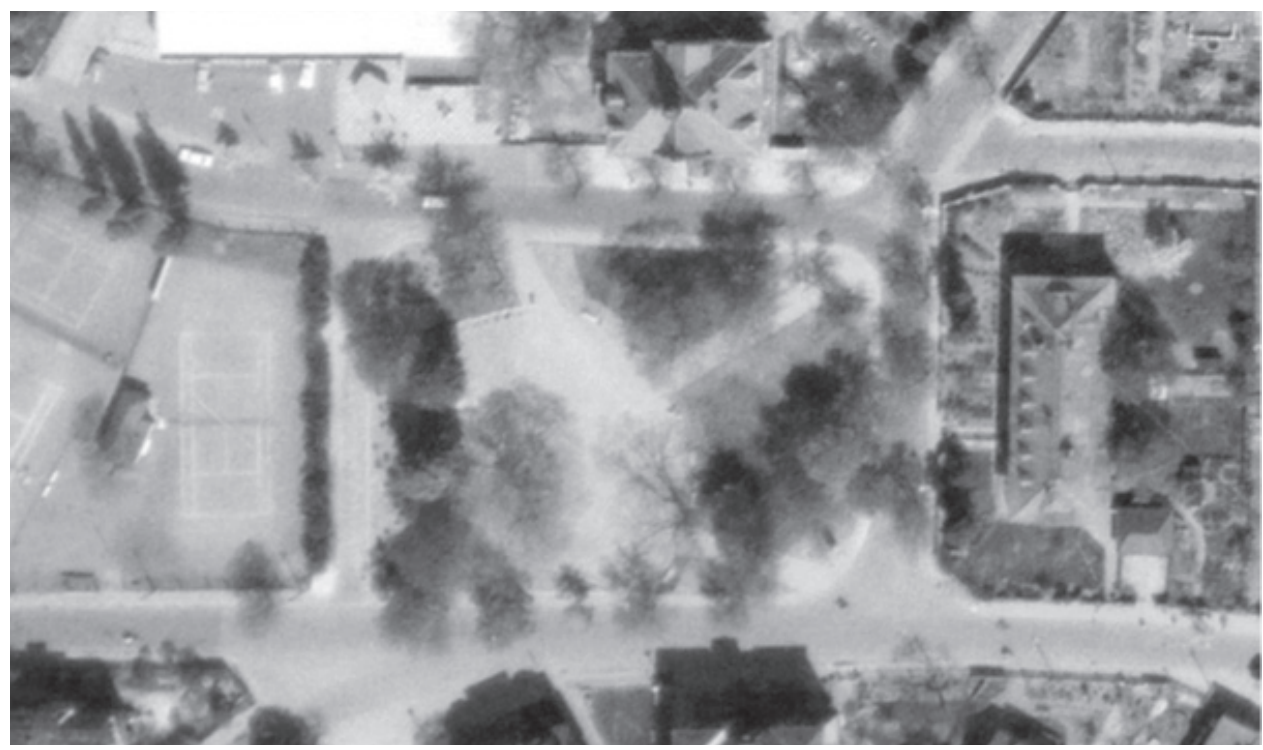

8 | Ausschnitt aus: Senkrechtluftbildaufnahme vom 6.5.1976 (Bundesarchiv Berlin, Bild 180 31/76/133).

Den vorhandenen Baumbestand integrierte Kretzschmar in die Planung; so bildet die Rot-Eiche (Quercus rubra) auf dem Podest eine gestalterische Einheit mit Spruchwand und Figurengruppe (Abb. 11). Die von Kretzschmar und Peschel vorgelegte Planung wurde allerdings erst 1985 bestätigt. Der neu gesetzte Termin für die Einweihung war der 8. Mai 1987 - aber auch dieser Termin konnte nicht eingehalten werden, da der bestellte Sandsteinblock für die Figurengruppe nicht rechtzeitig geliefert wurde. Lieferprobleme gab es auch beim Material für die Podeststufen, die Sockel, Einfassungsborde und Pflanzgefäße aus Mühlleitner Sandstein. Dies sorgte für weitere Verzögerungen bei der Fertigstellung. Die Gestaltung erfolgte wegen des Materialmangels später mit (Vor-Ort)Waschbeton. Im September 1988 konnte der umgestaltete Platz mit dem Peschel-Ehrenmal fertig gestellt werden. Die Gestaltung von Kretzschmar und Peschel, vor allem die Figurengruppe, wurde von mehreren Seiten kritisiert. Besonders scharf äußerte sich der Radebeuler Bildhauer Prof. Walter Howard, Mitglied im Kreiskomitee der Antifaschistischen Widerstandskämpfer Dresden Land:
»Die Gesamtanlage ist unwürdig. Vor allem die Sandsteingruppe von dem Bildhauer Johannes Peschel ist eine misslungene Arbeit. Diese Gruppe wird von der Bevölkerung kritisch beurteilt und abgelehnt. Das Kreiskomitee fordert das Wegräumen. [...] Ohne das Hinzuziehen des Komitees der Widerstandskämpfer und des 1980 berufenen Aktivs Bildender Künstler Radebeul hat der damalige Stadtrat für Kultur Meissner dieses Denkmal und die Gesamtanlage errichten lassen. $\ll^{23}$

Die Figurengruppe und die Spruchwand wurden 1990 abgebaut, zunächst zwischengelagert und dann auf dem Gelände der ehemaligen Radebeuler Waldparkschule abgestellt. Ob alle 15 Teile der Spruchwand vorhanden sind, ließ sich nicht genau ermitteln. Die Sandsteinfigur ist bis auf einige kleinere Beschädigungen in einem guten Zustand.

\section{Phase 5: Umgestaltung nach der Wende (1991 - 2017)}

Zwischen 1991 und 1993 erfolgte die denkmalgerechte Sanierung des Rathausgebäu- 


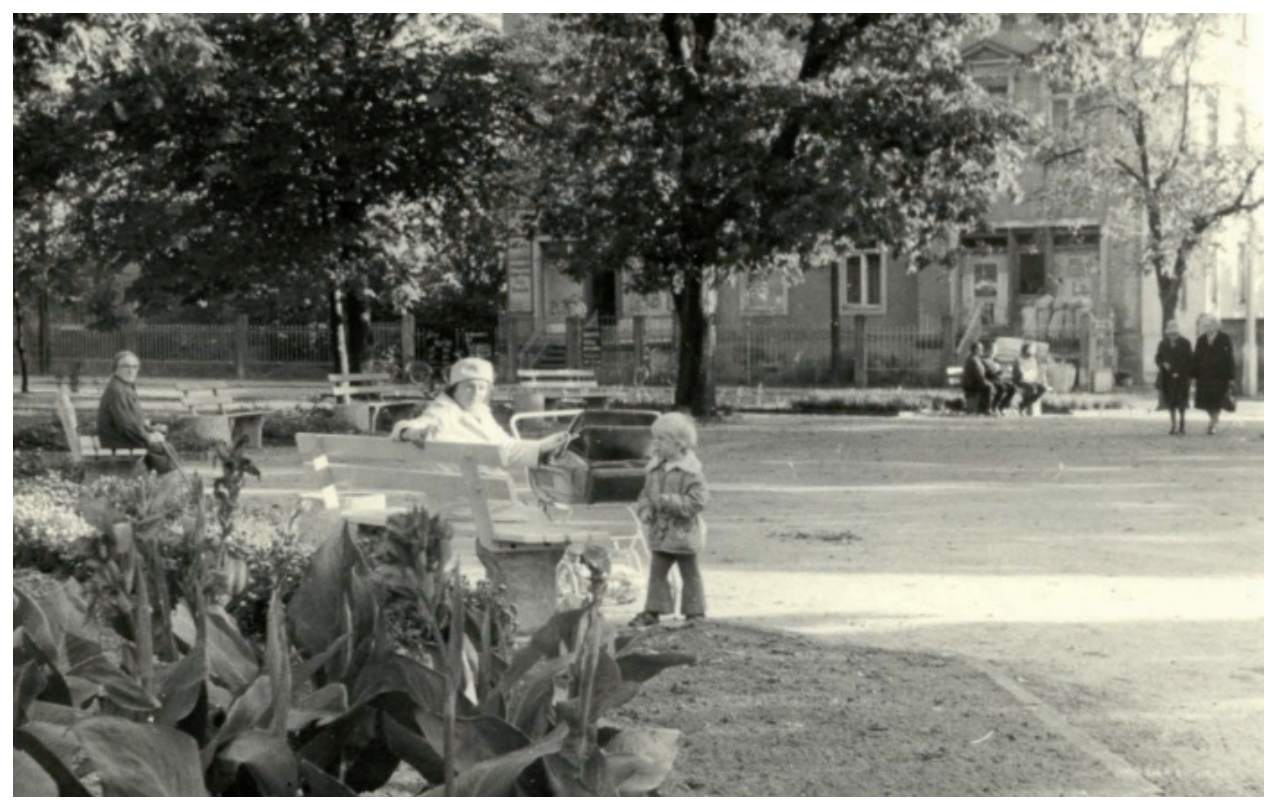

9 | Platz nach der Neugestaltung, Bankstandorte am halbkreisförmigen Weg, Blick nach Süden, Fotografie: Wolf Erhard, 18.10.1975 (Stadtarchiv Radebeul, Bild-Nr. 6981).

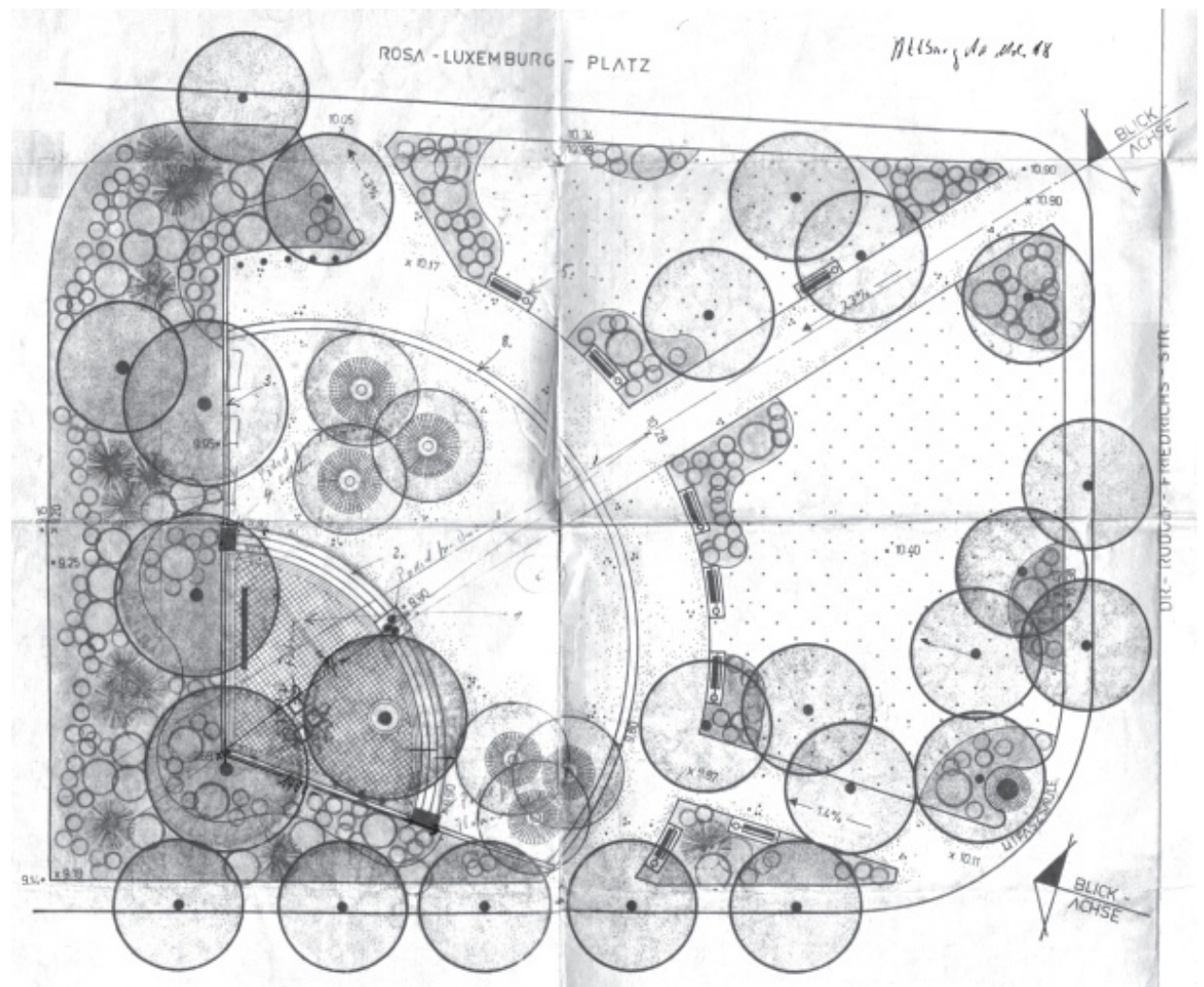

10 Entwurfskonzeption 1, Gestalterkollektiv Johannes Peschel, August 1985 (Stadtarchiv Radebeul, Akte DDR2992-VVN Denkmal gesamt). 
des $^{24}$ und ab Mitte Mai 1993 die Umnutzung zum Standesamt. Der Rosa-LuxemburgPlatz erhielt nun wieder eine repräsentative Funktion und war Kulisse für Hochzeitsfotos. Die Bänke wurden ausgetauscht und entsprachen nun dem Vorbild aus der Schmuckplatzzeit. Im Bereich vor dem Podest legte man zwei kleinere Rasenflächen mit mittigen Strauchpflanzungen an und pflanzte einige Gehölze innerhalb der Anlage. Die Staudenplanzungen wurden erneuert und die Kiesdecke auf dem Podest ausgebessert. Es erfolgte außerdem die Neupflanzung von drei Straßenbäumen an der Südseite und einem an der Ostseite. Am Standort der Spruchwand montierte man an einer schlichten, verputzten Betonstele die Granitplatte des VVN-Denkmals aus dem Jahr 1949. Neben der Betonstele stellte man zwei rechteckige Pflanzkübel aus Waschbeton auf. Der Platz der Figurengruppe auf dem mittleren Podest blieb frei, so dass weiterhin Gedenkveranstaltungen abgehalten werden konnten.

Im August 1990 beschloss die Stadtverordnetenversammlung Radebeul, dass die Stadtverwaltung einen Wettbewerb zur Gestaltung des Rosa-Luxemburg-Platzes als Radebeuler Gedenkstätte mit dem Thema »Für die Opfer und Gegner von Gewalt" vorbereiten sollte. ${ }^{25}$ Dieser Beschluss wurde aufgrund der finanziellen Belastung in Höhe von mindestens 30.000 DM kurze Zeit später wieder aufgehoben und der Wettbewerb zurückgezogen. Die Gestaltung sollte mit eigenen Planungskapazitäten umgesetzt werden. Als vorläufige Lösung wählte man eine kurzfristig realisierbare Variante: Weitere sechs Bänke sollten aufgestellt werden, die Fahnenmasten entfernt, zwei halbrunde Sommerblumenbeete angelegt und Papierkörbe sowie drei größere Pflanzschalen auf den leeren Sockelbereichen aufgestellt werden. Bis zur Festlegung des zukünftigen Platzcharakters sollte dieser in seinem jetzigen, wenn auch unbefriedigenden gestalterischen Zustand mit geringfügigen Änderungen erhalten bleiben. Die generelle Umgestaltung sollte erfolgen, sobald die erforderlichen Mittel dafür bereit stünden. ${ }^{26} 2007$ lobte die Stadt Radebeul den Wettbewerb für die Umgestaltung des Rosa-LuxemburgPlatzes neu aus. Die Aufgabenstellung veränderte sich völlig, so dass nur der Rückbau der Straße und dadurch folgend die Vergrößerung der Grünanlage, der Rückbau des Sockels mit Denkmal, die Erhaltung des Gedenksteins für die Opfer des Nationalsozialismus und die weitestgehende Erhaltung des Baumbestandes übrig blieben. ${ }^{27}$ Im Juli 2008 reichte das Dresdner Landschaftsarchitekturbüro Haufe Lohse Pätzig einen Entwurf zur Umgestaltung ein. Dieser stieß zunächst auf Zustimmung im Stadtrat, dem Bauausschuss waren allerdings die veranschlagten Kosten von 100.00o DM zu hoch und die Umgestaltung verschob sich auf unbestimmte Zeit. ${ }^{28}$ Auch viele Radebeuler äußerten sich in Leserbriefen kritisch zum geplanten Umbau. ${ }^{29}$ Ein knappes Jahrzehnt blieb der Rosa-Luxemburg-Platz unverändert bis 2017 die Umgestaltung neu diskutiert und die Ausführung des Entwurfes von Haufe Lohse Pätzig beschlossen wurde.

\section{Umgestaltung (ab 2018)}

Im Frühjahr 2018 wurde mit der Umgestaltung begonnen. Der Entwurf sieht einen »heiter gestalteten Platz« mit Verbesserung der Aufenthalts- und Durchgangsqualität durch eine zeitgemäße Formensprache vor (Abb. 12). Die Vegetation, die größtenteils erhalten wird, soll den großzügigen Platzcharakter bestimmen und der Gedenkstein auf würdige Weise platziert werden..$^{30}$ Das Podest sowie die Bepflanzungen im Inneren des Platzes werden entfernt. Zwei ineinander verdrehte, elliptische Formen ergeben einen Rasenspiegel im Zentrum der Anlage, um den eine neue Wegeführung aus wassergebundener Wegedecke führt. Lange Staudenbeete und Sitzbänke sind dieser Form angepasst. Zwei gepflasterte Hauptachsen an den Außenseiten schneiden die Ellipse in nordsüdlicher Richtung. Die Gedenktafel wird an die Westseite gesetzt.

Im Oktober 2018 waren die landschaftsbaulichen Arbeiten auf dem Platz weitestge- 


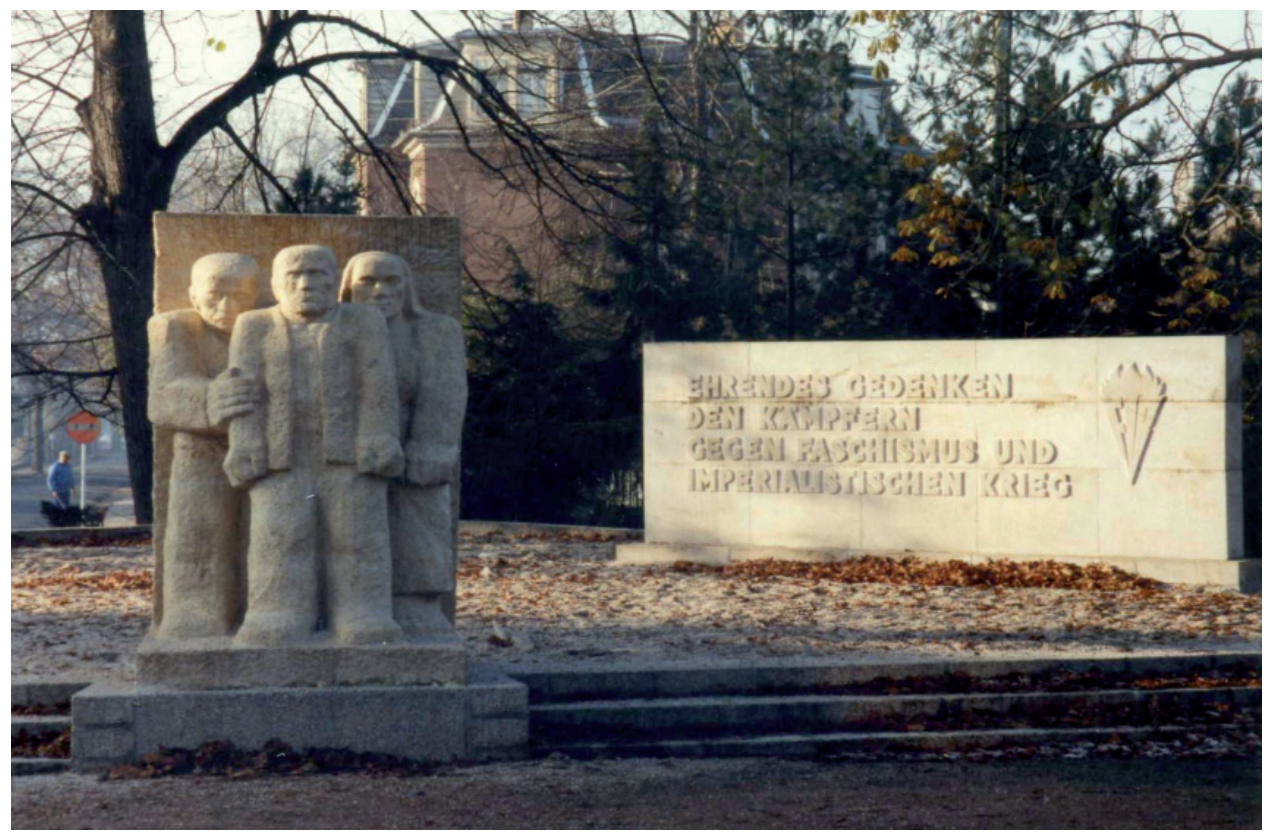

11 | Frontaler Blick auf Figurengruppe mit Spruchwand, Fotografie, 8.11.1989 (https://www.flickr.com/photos/ sludgeulper/4080646089/in/photolist-7dAnV4, Stand: 10.2.2018).

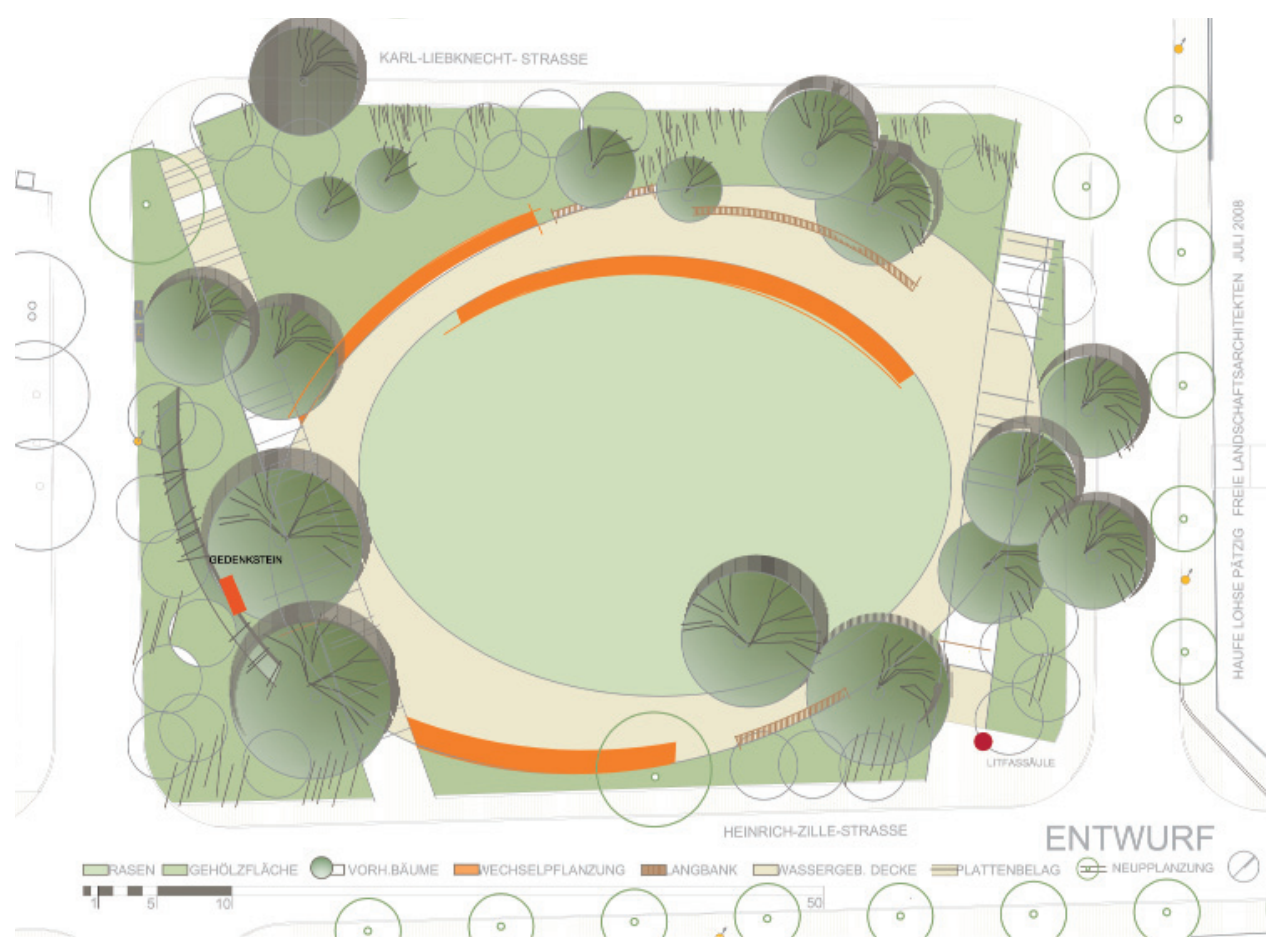

12 | Entwurfsplan Büro Haufe Lohse Pätzig Landschaftsarchitekten, Juli 2008. 


\section{Der Rosa-Luxemburg-Platz in Radebeul}

hend abgeschlossen und zahlreiche Gehölze und Hecken gerodet. Die im Entwurf vorgesehenen neuen Stauden- und Heckenpflanzungen stehen noch aus. Es wird sich zeigen, wie die Bürger Radebeuls den Platz zukünftig nutzen und ob die zeitgemäße Umgestaltung dem Anspruch eines Gedenkortes weiterhin gerecht wird.
1 Meyer, Gustav: Lehrbuch der schönen Gartenkunst, Berlin 1873, S.10.

2 Dietze, Kerstin: Villengärten in Radebeul. Beiträge zur Stadtkultur der Stadt Radebeul, Radebeul 2001, S. 6.

3 Ebd., S.3.

4 Gebrüder Ziller, in: http://tourismus.radebeul.de/ tourismus-kultur/stadt-und-historie/persoenlichkeiten/moritz-ziller/ (Stand: 13.10.2018).

5 Alexander Steffen (1871-1954) absolvierte eine Ausbildung zum Hofgärtner im Großherzoglichen Hofküchengarten in Schwerin und in der Königlichen Gärtnerlehranstalt Potsdam-Wildpark. Danach war er in der Städtischen Gartenverwaltung Berlin und Magdeburg tätig, von 1895-1897 in der Baumschule und Landschaftsgärtnerei „E. Pietzsch“ in Oberlößnitz. Zu dieser Zeit wirkte er am Königsplatz. 1922-1936 war er Gartendirektor der staatlichen Versuchs- und Beispielgärtnerei Pillnitz bei Dresden, vgl. hierzu: Gröning, Gerd/ WolschkeBulmann, Joachim: Grüne Biographien: Biographisches Handbuch zur Landschaftsarchitektur des 20. Jahrhunderts in Deutschland, Berlin 1997, S. 378.

6 Stadtarchiv Radebeul, NL-5069-Königsplatz, Beschluss der Königlichen Amtshauptmannschaft Dresden-Neustadt vom 19.5.1894.

7 Altner, Manfred: Stadtlexikon Radebeul. Historisches Handbuch für die Lößnitz, Radebeul 2006, S. 257.

8 Stadtarchiv Radebeul, NL-5070-Königsplatz, Blatt 10 Kostenanschlag von Pietzsch.

9 Ebd., Schreiben vom Verschönerungsverein an den Gemeinderat vom 29.6.1895.

10 Ebd., Schreiben von Gustav Pietzsch an den Gemeindevorstand Herz vom 26.7.1895.

11 Ebd., Protokollauszug aus der Gemeinderatsitzung vom 9.3.1899.

12 Stadtarchiv Radebeul, NL-5069-Königsplatz, Auszug aus dem Sitzungsprotokoll des Bauausschusses vom 9.3.1915.

13 Der exakte Standort des Kaiser-Wilhelm-Denkmals ist nicht dokumentiert. Allerdings weist das Luftbild aus dem Jahr 1945 darauf hin, dass das Denkmal zwischen Wasserbassin und Rathaus in Blickbeziehung zur Friedensburg stand. Diese Blickbeziehung bestand auch schon am ursprünglichen Standort des Denkmals an der Kreuzung Königstraße / Kaiserstraße.

14 Stadtarchiv Radebeul, NL-5069-Königsplatz, Blatt 1. 15 Täubert, Gudrun/ Andert, Frank: Schmuckplätze in Radebeul gestern und heute, Radebeul 2010, S. 32.

16 Stadtarchiv Radebeul, S 11-21 Gedenkstätten der Radebeuler Arbeiterbewegung, Blatt 2.

$17 \mathrm{Ob}$ und wann diese Gestaltung umgesetzt wurde, ließ sich aufgrund fehlender Bildquellen nicht mit absoluter Sicherheit feststellen. Auch die Akten im Stadtarchiv Radebeul gaben keinen Aufschluss darüber, welche Arbeiten von der VVN und anderen Akteuren nach 1950 auf dem Rosa-LuxemburgPlatz ausgeführt wurden.

18 Altner 2006, S. 162.

19 Stadtarchiv Radebeul, DDR-2992, VVN-Denkmal Rosa-Luxemburg-Platz, Brief der SED Ortsparteiorganisation Radebeul an den Rat der Stadt Radebeul vom 16.10.1979.

20 Ebd., Brief des Stadtrates für Kultur Radebeul Meiner an J. Peschel vom 2.9.1980.

21 Ebd, Erläuterungsbericht zur Neugestaltung VdNDenkmal Rosa-Luxemburg-Platz-Radebeul vom 17.8.1985.

22 Abmaße 1,50 x 1,50 x 1,80 m.

23 Stadtarchiv Radebeul, DDR-2992, VVN-Denkmal Rosa-Luxemburg-Platz, Brief von Prof. Walter Howard an den Rat der Stadt Radebeul, 16.9.1989.

24 Altner 2006, S. 162.

25 Stadtarchiv Radebeul, DDR-2992-VVN Denkmal, Beschlussvorlage zu Beschluß-Nr. 20/90.

26 Ebd., Zusammenfassender Bericht der Stadtverwaltung Radebeul, Bauverwaltung, Abt. Stadtgrün zur geplanten Umgestaltung des Rosa-LuxemburgPlatzes vom 1.3.1991.

27 Heike Funke, Mitarbeiterin Stadt Radebeul, Amt für Stadtgrün, E-Mail vom 15.12.2017.

28 Berndt, Henry: Planung für Rosa-Luxemburg-Platz fällt durch, Sächsische Zeitung Radebeul, 4.9.2008.

29 Berndt, Henry: SZ-Leser wollen keinen neuen Luxemburgplatz, Sächsische Zeitung Radebeul, 13./14.09.2008.

30 Stadt Radebeul, Amt für Stadtgrün, Unterlagen zur Entwurfsplanung vom Landschaftsarchitekturbüro Haufe Lohse Pätzig, Juli 2008. 